\title{
Reduced interhemispheric transmission in schizophrenia patients: evidence from event-related potentials
}

\author{
Tanja Endrass ${ }^{a, *}$, Bettina Mohr ${ }^{a, b}$, Brigitte Rockstroh ${ }^{a}$ \\ ${ }^{a}$ Department of Psychology, University of Konstanz, PO-Box D23, 78457 Konstanz, Germany \\ ${ }^{b}$ Medical Research Council, Cognition and Brain Sciences Unit, 15 Chaucer Road, Cambridge CB2 2EF, UK
}

Received 24 September 2001; received in revised form 28 December 2001; accepted 28 December 2001

\begin{abstract}
Interhemispheric transfer was investigated in 14 schizophrenia patients and 15 age- and sex-matched healthy controls in a lateralized lexical decision task. Words and pseudowords were tachistoscopically presented either to the left or to the right visual hemifield. Event-related potentials were determined from a 65-channel electroencephalogram. Information transfer between hemispheres was assessed by the interhemispheric transmission time (IHTT), the N1-latency difference between ipsilateral and contralateral hemisphere.

Controls, but not schizophrenia patients showed significantly faster IHTT from the right to the left hemisphere for words, while IHTT from the left to the right hemisphere did not differ between groups and stimuli. These findings are interpreted in terms of a deficit in schizophrenia to transfer verbal information from the right to the left hemisphere via the corpus callosum. (C) 2002 Elsevier Science Ireland Ltd. All rights reserved.
\end{abstract}

Keywords: Schizophrenia; Interhemispheric transmission; Lexical decision; Hemispheric asymmetry; Corpus callosum; Interhemispheric transmission time

Alterations of hemispheric functions as well as dysfunctional information transfer between the two cerebral hemispheres have been described for schizophrenia disorders $[1,4]$. While there is empirical support for subtle alterations of brain structures in the left hemisphere ( $\mathrm{LH})$, in particular abnormalities of the superior temporal gyrus in schizophrenia patients [6], many studies report normal functional lateralization during cognitive processing [11,13,14]. In contrast, the empirical evidence for structural deviations of the corpus callosum (reduced fibre density or more generally a reduction in corpus callosum size) $[8,18]$ and functional impairments in interhemispheric transmission $[1,5,7,13]$ seems to be more consistent.

In addition to behavioural performance, interhemispheric transfer can be measured even more precisely, by the interhemispheric transmission time (IHTT) in the electroencephalogram (EEG). The IHTT is assessed in studies employing visual hemifield stimulation and is defined as the latency difference of early-occurring evoked potential

\footnotetext{
* Corresponding author. Tel.: +49-7531-883-207; fax: +497531-882-891.

E-mail addresses: tanja.endrass@uni-konstanz.de

(T. Endrass), bettina.mohr@mrc-cbu.cam.ac.uk (B. Mohr), brigitte.rockstroh@uni-konstanz.de (B. Rockstroh).
}

components between the contralateral and the ipsilateral hemisphere $[2,3,9,15]$. Brown et al. [3] stated in their meta-analysis of event-related potential (ERP) studies, that IHTT in most verbal and non-verbal studies is shorter after stimulus presentation to the left visual hemifield (LVF), when information travels from the right hemisphere (RH) to the LH [9], which reflects a directional asymmetry of callosal transmission. Independent of task specific cerebral dominance, these data can be explained by asymmetries of callosal fiber connections between hemispheres with a greater number of neurons projecting from the $\mathrm{RH}$ to the LH, than vice versa [12]. Nowicka et al. [15] found directional asymmetry to be dependent on functional laterality, which means faster transmission from the non-specialized hemisphere to the task-specific advantaged hemisphere. Thus, there is some evidence for impaired interhemispheric transfer between the two cerebral hemispheres in schizophrenia. The present study examined interhemispheric transfer by analysing EEG correlates of language processing in schizophrenia patients.

Fifteen healthy controls (seven females; mean age: 23.9 years; education: 12.6 years; handedness score: 96.0 assessed by the Edinburgh Handedness Inventory [16]) and 14 schizophrenia patients (five females; mean age: 


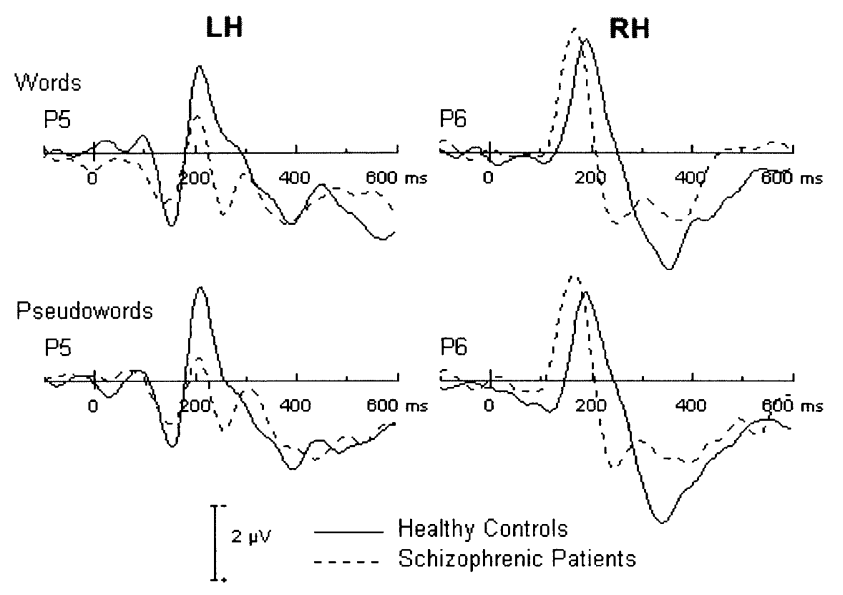

Fig. 1. ERPs at parietal electrodes P5 (LH) and P6 (RH) after LVF stimulation for words (upper part) and pseudowords (lower part) separately for healthy controls (black line) and for schizophrenic patients (dashed line).

27.3 years; education: 11.2 years; handedness score: 97.1) were paid for study participation. All subjects were native speakers of German and right-handed. Patients were recruited from the Center of Psychiatry, Reichenau and met DSM-IV criteria of paranoid schizophrenia. Patients received antipsychotic medication with standard neuroleptic drugs (Chlorpromacine equivalent: $293 \mathrm{CPZ}$ ). Their mean Brief Psychiatric Rating Scale (BPRS)-Score [10] was 45.5. One-way analyses of variance (ANOVA) found no significant group differences for the variables age, education and handedness.

In a lexical decision task participants were instructed to decide whether a visually presented letter string was a meaningful German word or a meaningless pseudoword by bimanually pressing two out of four response buttons. Stimuli were presented tachistoscopically for $150 \mathrm{~ms}$ either unilaterally to the right visual field (RVF) or to the LVF or simultaneously to both visual fields (BVF). The inter-stimulus-interval varied between 2.5 and $3.5 \mathrm{~s}$. Subjects were instructed to respond as quickly and as correctly as possible. A 60 trials practice block preceded the experiment comprising 240 stimuli (120 words and 120 pseudowords) presented in pseudo-randomised order. Words and pseudowords were presented in black upper-case letters on a grey background to reduce visual afterimages. All stimuli comprised two syllables and were four to eight letters long. Words were German content words with a high frequency of occurrence (1000-10 000 occurrences per 10 million words, according to Ortmann [17]). Pseudowords were obtained either by permutation of letters within or by exchanging letters between word stimuli. Pseudowords were pronounceable and orthographically regular but not homophonous to real words. All items were presented on a 17-inch monitor of an IBM compatible Pentium PC placed at a distance of one meter in front of the participants. Stimuli appeared between $1.5^{\circ}$ and $4.5^{\circ}$ of horizontal and $0.6^{\circ}$ of vertical visual angle for $150 \mathrm{~ms}$. During the experiment, all participants had their

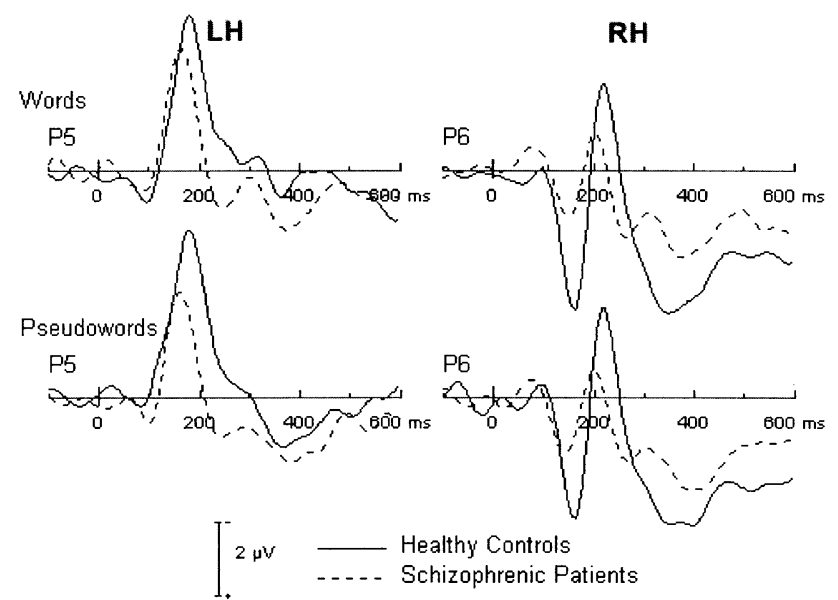

Fig. 2. ERPs at P5 and P6 after RVF stimulation with words (upper part) and pseudowords (lower part) separately for healthy controls (black line) and for schizophrenic patients (dashed line).

chins in a chin rest with a forehead restraint bar centred relative to the viewing screen.

The EEG was recorded with two 32-channel DC-amplifiers (SYNAMPS) from 64 head positions referenced to CZ using an electrode cap (Electrocap Inc.). Signals were digitised with a bandpass of $0.1-30 \mathrm{~Hz}$ and at $200 \mathrm{~Hz}$ sampling rate. The electrooculogram was recorded for later artifact correction. Only artifact free trials for correct responses within $2 \mathrm{~s}$ after stimulus onset were averaged for analysis. The number of trials included in the data analysis did not differ significantly between groups. First, the N1-latency was calculated by determining the most negative peak during the time window from 100 to $300 \mathrm{~ms}$. As in previous studies [2] parietal electrode sites were selected because the N1-amplitude was most pronounced over these cortical areas. Over each hemisphere, three parietal electrodes (P5, P7 and PO7 for LH and P6, P8 and PO8 for RH) were selected. IHTT refers to the latency difference between ipsilateral and contralateral N1-latency. Statistical analysis was done for the N1-latency and for the IHTT.

Effects for errors and latencies were evaluated by ANOVAs with the factors Group, Visual Field (LVF versus RVF) and Wordness (Word versus Pseudoword). The significant Visual Field $\times$ Wordness interaction (errors: $F_{1,27}=5.1, P<0.04$; latencies: $F_{1,27}=6.4, P<0.02$ ) indicated a stronger RVFA for words than for pseudowords. As indicated by the main effect Group, schizophrenia patients made significantly more errors $\left(F_{1,27}=9.1\right.$, $P<0.02)$ and were significantly slower to respond $\left(F_{1,27}=5.7, P<0.03\right)$. No significant Group $\times$ Visual Field $\times$ Wordness interaction was obtained neither for correct responses nor for reaction times.

Figs. 1 and 2 illustrate the time course of the ERPs at parietal sites over the LH and RH, following LVF and RVF stimulation. Effects of the N1-latencies were obtained from the ANOVA with the factors Group, Wordness (Words versus Pseudowords), Visual Field (LVF versus RVF) and 
Hemisphere (LH versus RH). The significant main effect for Group $\left(F_{1,27}=7.1, P<0.02\right)$ indicated shorter overall N1latencies for patients compared to controls. The Visual Field $\times$ Hemisphere interaction $\left(F_{1,27}=160.7, P<0.01\right)$ refers to shorter contralateral than ipsilateral N1-latencies over the LH after RVF stimulation and over the RH after LVF stimulation. As revealed by the Group $\times$ Hemisphere interaction $\left(F_{1,27}=10.2 ; P<0.01\right)$, schizophrenia patients displayed shorter N1-latencies than controls only over the $\mathrm{RH}$, but not over the LH. A significant Wordness $\times$ Visual Field $\times$ Hemisphere interaction $\left(F_{1,27}=5.1 ; P<0.04\right)$ was obtained, planed comparison revealed, that after LVF stimulation over the RH, pseudowords had significantly shorter N1-latencies than words $\left(F_{1,27}=6.5 ; P<0.02\right)$. For analysing IHTT, an ANOVA with the factors Group, Wordness and Visual Field revealed a significant main effect for Wordness $\left(F_{1,27}=5.1 ; \quad P<0.04\right)$ and a significant Wordness $\times$ Visual Field interaction $\left(F_{1,27}=4.6\right.$; $P<0.05)$, with increased IHTT for pseudowords after LVF stimulation. A significant Group $\times$ Visual Field interaction $\left(F_{1,27}=9.1 ; P=0.006\right)$ demonstrated, that in controls IHTT after LVF stimulation was shorter than after RVF stimulation, and controls had shorter IHTT than patients in the LVF condition. In contrast, transmission times did not differ significantly between the two visual fields in schizophrenia patients. Since no significant threeway interaction was found in this analysis, ANOVAs on IHTT were calculated separately for controls and patients. Only in the control group a significant Wordness $\times$ Visual Field interaction $\left(F_{1,14}=4.6 ; P<0.05\right)$ was found, with shorter IHTT for words after LVF stimulation. As expected after simultaneous bilateral stimulation, N1-latencies as well as IHTT did not differ between hemispheres, wordness and groups in the bilateral condition (Fig. 3).

In the behavioural data, a clear RVFA for words, that is, left-hemispheric superiority for this task was found. The RVFA in both groups supports previous findings which

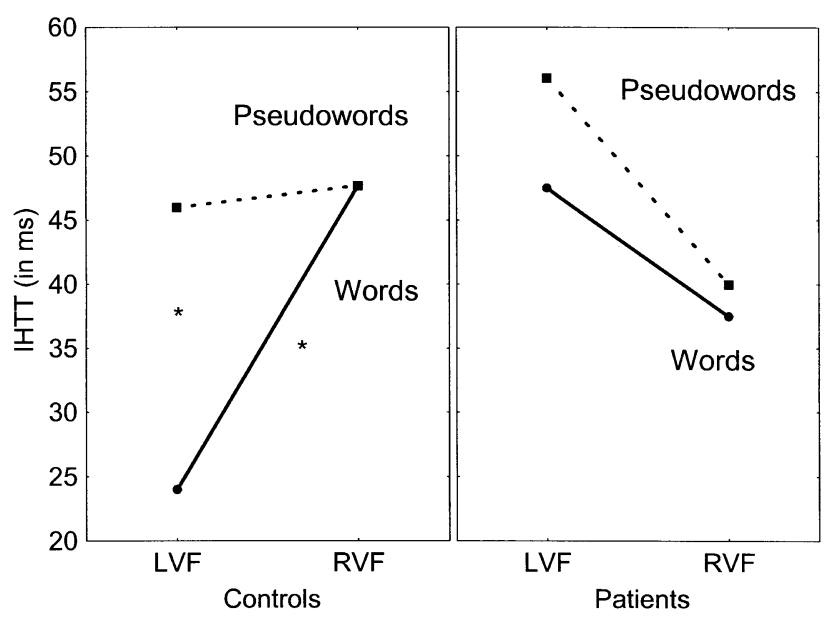

Fig. 3. Mean IHTT (in ms) for words and pseudowords is presented separately for the control and patient group and for the LVF and the RVF. found normal language lateralization in schizophrenia patients $[13,14]$. The analysis of the N1-latency showed a significant Visual Field $\times$ Hemisphere interaction, indicating shorter N1-latencies in the initially stimulated contralateral hemisphere and longer latencies in the ipsilateral hemisphere which received the input later in time. A delayed activation of the ipsilateral hemisphere points to the transfer of information via the corpus callosum originating from the initially activated contralateral hemisphere $[3,9,15]$. In addition, we found a group particularly over the RH indicated by the Group $\times$ Hemisphere interaction, with patients exhibiting significantly shorter N1-latencies over the RH than controls. The longer N1-latencies over the RH in controls may result from a directional asymmetry of hemispheric transmission, with faster transmission from the RH to the LH during word processing, which is less attenuated in patients. This hypothesis is supported by the Group $\times$ Visual Field interaction of IHTT, which found increased IHTT after LVF stimulation in schizophrenia patients compared to controls. The Wordness $\times$ Visual Field interaction of IHTT demonstrated, that after LVF stimulation, IHTT was only reduced for words, but not for pseudowords. For controls, a significant difference between words and pseudowords in IHTT was found. It turned out that only words caused a directional asymmetry but transmission time of pseudowords did not differ between the two visual fields $[3,9,15]$. In patients no such difference could be found. This result supports the findings from Novicka et al. [15] in which IHTT from RH to LH was increased for words. According to these authors, only for words a LH dominance exists and therefore a faster transmission from the RH to the LH would be expected. In our study, the directional asymmetry in controls was congruent with the visual field advantage: words elicited a larger RVFA and shorter IHTT from RH to LH whereas pseudowords (no LH dominance) elicited a smaller RVFA and no IHTT difference between the two visual field conditions.

For schizophrenia patients, interhemispheric transmission in both directions took the same time for words and pseudowords. After LVF stimulation, shorter N1-latencies over RH was found in patients. Although the ipsilateral N1 amplitudes seemed to be reduced in patients, statistical analyses did not confirm this effect. There are basically two possible explanations for the present findings. The reduced directional asymmetry of IHTT for words in patients could be caused by the reduced ability of the RH to differentiate between words and pseudowords or by the difficulty to detect meaningful material. This less efficient pseudoword processing by the $\mathrm{RH}$, which may be time consuming, may prevent a fast transmission of words to the $\mathrm{LH}$ via the corpus callosum. The second explanation of the data could be that there is a deficit of the corpus callosum in schizophrenia patients which leads to less efficient and longer information transfer between the two hemispheres. Behavioural results from our earlier studies $[13,14]$ support the view of impaired interhemispheric transmission and rather speak against the 
interpretation of a right hemispheric deficit in processing verbal material.

However, more research is needed to find an adequate explanation for the observed alterations of IHTT from the $\mathrm{RH}$ to the LH in schizophrenia patients. In particular, it is still unclear whether the transmission deficit is only related to language stimuli or if it also occurs for non-verbal stimuli for which a RH specialization can be assumed.

We would like to thank Gabriele Geiger and Katja Hannemann for help in conducting the experiment and Dr Hans Watzl and Dipl.-Psych. Patricia Roessner for help in patient recruitment. Supported by grants Mo 697/1-1, Mo 697/2-1 and Ro 805/11-1 of the Deutsche Forschungsgemeinschaft and by grant AFF 8/99 of the University of Konstanz.

[1] Beaumont, J.G. and Dimond, S.J., Brain disconnection and schizophrenia, Br. J. Psychiatry, 123 (1973) 661-662.

[2] Brown, W.S. and Jeeves, M.A., Bilateral visual field processing and evoked potential interhemispheric transmission time, Neuropsychologia, 31 (1993) 1267-1281.

[3] Brown, W.S., Larson, E.B. and Jeeves, M.A., Directional asymmetries in interhemispheric transmission time: evidence from visual evoked potentials, Neuropsychologia, 32 (1994) 439-448.

[4] Crow, T.J., Schizophrenia as a failure of hemispheric dominance for language, Trends Neurosci., 20 (1997) 339-343.

[5] David, A.S., Tachistoscopic tests of colour naming and matching in schizophrenia: evidence for posterior callosum dysfunction? Psychol. Med., 17 (1987) 621-630.

[6] DeLisi, L.E., Sakuma, M., Kusher, M., Finer, D.L., Hoff, A.L. and Crow, T.J., Anomalous cerebral asymmetry and language processing in schizophrenia, Schizophr. Bull., 23 (1997) 255-271.

[7] Dimond, S.J., Scammell, R., Pryce, I.J., Huws, D. and Gray, C., Some failures of intermanual and cross-lateral transfer in chronic schizophrenia, J. Abnorm. Psychol., 89 (1980) 505-509.

[8] Highley, J.R., Esiri, M.M., McDonald, B., Roberts, H.C., Wakler, M.A. and Crow, T.J., The size and fiber composition of the anterior commissure with respect to gender and schizophrenia, Biol. Psychiatry, 45 (1999) 1120-1127.

[9] Larson, E.B. and Brown, W.S., Bilateral field interactions, hemispheric specialization and evoked potential interhemispheric transmission time, Neuropsychologia, 35 (1997) 573-581.

[10] Lukoff, D., Nuechterlein, K., Ventura, J. and Appendix, A., Manual for expanded brief psychiatiric rating scale (BPRS), Schizophr. Bull., 12 (1986) 594-602.

[11] Magaro, P.A. and Chamrad, D.L., Information processing and lateralization in schizophrenia, Biol. Psychiatry, 18 (1983) 29-44.

[12] Marzi, C.A., Bisiacchi, P. and Nicoletti, R., Is interhemispheric transfer of visuomotor information asymmetric? Evidence from a meta-analysis, Neuropsychologia, 29 (1991) 1163-1177.

[13] Mohr, B., Pulvermüller, F., Cohen, R. and Rockstroh, B., Interhemispheric cooperation during word processing: evidence for callosal transfer dysfunction in schizophrenic patients, Schizophr. Res., 46 (2000) 231-239.

[14] Mohr, B., Functional asymmetry in schizophrenic patients during auditory speech processing, Schizophr. Res., (2001) 52.

[15] Nowicka, A., Grabowska, A. and Fersten, E., Interhemispheric transmission of information and functional asymmetry of the human brain, Neuropsychologia, 34 (1996) 147-151.

[16] Oldfield, R.C., The assessment and analysis of handedness: the Edinburgh inventory, Neuropsychologia, 9 (1971) 97113.

[17] Ortmann, W.D., Hochfrequente Deutsche Wortformen, Bde. 1 \& 2, Goethe-Institut, Arbeitsstelle für Wissenschaftliche Didaktik, München, 1975.

[18] Woodruff, P.W.R., Phillips, M.L., Rushe, T., Wright, I.C., Murray, R.M. and David, A.S., Corpus callosum size and inter-hemispheric function in schizophrenia, Schizophr. Res., 23 (1997) 189-196. 